\title{
Paving the way for TNF vaccines
}

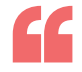 \\ The onset of arthritis was delayed by 15 days and its prevalence was halved}

A novel TNF vaccine that uses diphtheria toxin as a molecular scaffold delays disease onset and dramatically reduces arthritis severity in a mouse model of rheumatoid arthritis, according to new research published in PLOS ONE. "This is the first report of vaccine design [being] based on a clinical[ly] safe carrier and scaffold," reports Rongxiu Li, corresponding author on the study.

Despite showing promise in animal models of disease, previous candidate TNF vaccines have lacked efficacy when trialled in humans. $\mathrm{Li}$ and colleagues hypothesized that by utilizing a protein currently in use as a vaccine as a scaffold for the TNF peptide, they could reduce the toxicity and increase the stability of the final vaccine. "[The transmembrane domain of diphtheria toxin] is structurally stable [owing] to its alpha-helix and loop composition, possesses universal ... epitopes [recognized by helper T cells,] essential for breaking T-cell tolerance, and is highly soluble when overexpressed in E. coli," explians Li.

The researchers tested their TNF vaccine in mice with collagen-induced arthritis. The vaccine was administered 2 weeks after inducing disease. The onset of arthritis was delayed by 15 days and its prevalence was halved ( $\sim 90 \%$ versus $\sim 45 \%$ ) in mice that received the diphtheria toxin-TNF hybrid vaccine, compared with mice

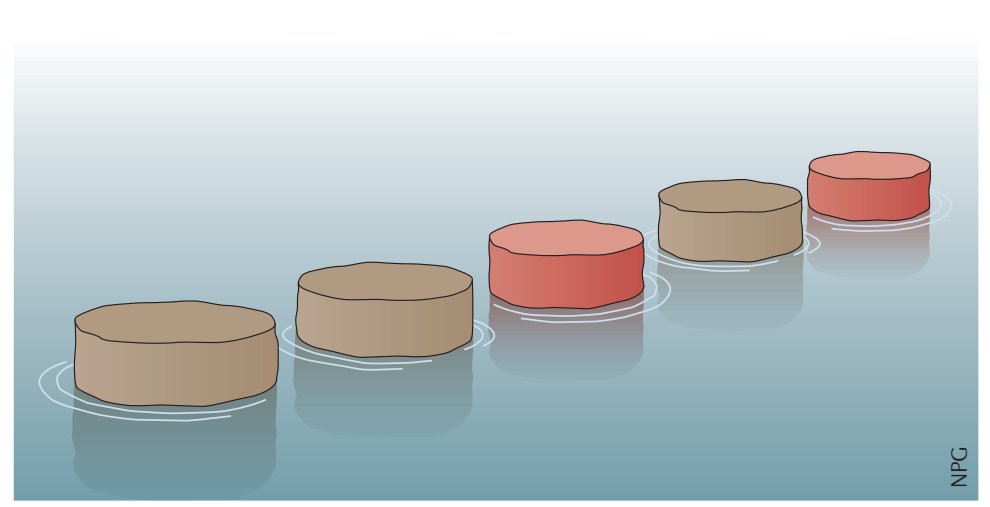

that received diphtheria toxin alone. Mice receiving the hybrid TNF vaccine also had an eightfold reduction in their clinical arthritis score, confirmed by histology as a reduction in bone erosion, synovial inflammation, immune cell infiltration and cartilage damage.

Interestingly, immunization of mice with the engineered TNF vaccine stimulated high titres of anti-TNF antibodies, the vast majority of which were of the IgG1 subtype, indicating that class-switching had taken place. "Remarkably, the antibody titre was sustained for more than 6 months, which is an advantage for [anti-TNF] therapy in [a chronic] setting such as rheumatoid arthritis," states Li. The sustained anti-TNF antibody titre is an improvement on previous candidate TNF vaccines, in which these antibody titres were usually short-lived.

The research team plan to continue trialling this vaccine in other preclinical models of rheumatoid arthritis and hope that it will one day reach the clinic.

Joanna Collison

ORIGINAL ARTICLE Zhang, L. et al. A rationally designed TNF- $\alpha$ epitope-scaffold immunogen induces sustained antibody response and alleviates collagen-induced arthritis in mice. PLoS ONE 11, e0163080 (2016) 